\title{
Design, Synthesis and In Vitro Antimicrobial Activity of 6-(1H-Benzimidazol-2-yl)-3,5-dimethyl-4-oxo-2-thio-3,4- dihydrothieno[2,3-d]pyrimidines
}

\author{
Sergiy V. Vlasov ${ }^{1}$, Olena D. Vlasova ${ }^{1}$, Hanna I. Severina ${ }^{1} \mathbb{D}$, Konstantin Yu. Krolenko ${ }^{2} \mathbb{D}$, \\ Oleksandr V. Borysov ${ }^{2,3}$, Amjad Ibrahim M. Abu Sharkh ${ }^{1}$, Vitaliy S. Vlasov ${ }^{1}$ and Victoriya A. Georgiyants ${ }^{1, *(D)}$ \\ 1 Department of Pharmaceutical Chemistry, National University of Pharmacy, 53 Pushkinska St., \\ 61002 Kharkiv, Ukraine; sergiy.vlasov@gmail.com (S.V.V.); olenavlasova94@gmail.com (O.D.V.); \\ severina.ai@ukr.net (H.I.S.); amjad1977a@gmail.com (A.I.M.A.S.); vsv@nuph.edu.ua (V.S.V.) \\ 2 Enamine Ltd., 78 Chervonotkatska St., 02094 Kyiv, Ukraine; krolenko.ky@gmail.com (K.Y.K.); \\ boav.79@gmail.com (O.V.B.) \\ 3 Institute of Organic Chemistry, National Academy of Sciences of Ukraine, 5 Murmanska St., \\ 02660 Kyiv, Ukraine \\ * Correspondence: vgeor@ukr.net; Tel.: +38-050-084-18-71
}

\section{check for} updates

Citation: Vlasov, S.V.; Vlasova, O.D.; Severina, H.I.; Krolenko, K.Y.; Borysov, O.V.; Abu Sharkh, A.I.M.; Vlasov, V.S.; Georgiyants, V.A. Design, Synthesis and In Vitro Antimicrobial Activity of 6-(1H-Benzimidazol-2-yl)3,5-dimethyl-4-oxo-2-thio-3,4dihydrothieno[2,3- $d$ ]pyrimidines. Sci. Pharm. 2021, 89, 49. https://doi.org/ 10.3390/scipharm89040049

Academic Editor: Roman B. Lesyk

Received: 23 October 2021

Accepted: 16 November 2021

Published: 18 November 2021

Publisher's Note: MDPI stays neutral with regard to jurisdictional claims in published maps and institutional affiliations.

Copyright: (c) 2021 by the authors. Licensee MDPI, Basel, Switzerland. This article is an open access article distributed under the terms and conditions of the Creative Commons Attribution (CC BY) license (https:/ / creativecommons.org/licenses/by/ $4.0 /)$.

\begin{abstract}
The rapid development in bacterial resistance to many groups of known antibiotics forces the researchers to discover antibacterial drug candidates with previously unknown mechanisms of action, one of the most relevant being the inhibition of tRNA (Guanine37-N1)-methyltransferase (TrmD). The discovery of selective TrmD inhibitors in the series of carboxamide derivatives of thienopyrimidines became a background for further modification of the similar structures aimed at the development of promising antibacterial agents. As part of this research, we carried out the construction of heterocyclic hybrids bearing the moieties of thieno[2,3- $d$ ]pyrimidine and benzimidazole starting from 3,5-dimethyl-4-oxo-2-thioxo- $1 H$-thieno[2,3- $d$ ]pyrimidine-6-carboxylic acid, which was used as the pivotal intermediate. The hybrid molecule of 6-(1H-benzimidazol-2-yl)-3,5-dimethyl2-thioxo-1H-thieno[2,3- $d$ ]pyrimidin-4-one prepared via condensation of the carboxylic acid with ortho-phenylenediamine was further alkylated with aryl/hetaryl chloroacetamides and benzyl chloride to produce the series of $S$-alkyl derivatives. The results of molecular docking studies for the obtained series of $S$-alkyl benzimidazole-thienopyrimidines showed their high affinity to the TrmD isolated from the P. aeruginosa. The results of antimicrobial activity screening revealed the antimicrobial properties for all of the studied molecules against both Gram-positive and Gram-negative bacteria and the Candida albicans fungal strain. The highest antimicrobial activity was determined for 2-\{[6-(1H-benzimidazol-2-yl)-3,5-dimethyl-4-oxo-3,4-dihydrothieno[2,3-d]pyrimidin-2-yl]thio\}$N$-(4-isopropylphenyl)acetamide, which also had the highest affinity to the TrmD inhibitor's binding site according to the docking studies results.
\end{abstract}

Keywords: thieno[2,3- $d$ ]pyrimidine; antimicrobial activity; TrmD inhibitor; docking study; Pseudomonas aeruginosa

\section{Introduction}

Recently, the wide spread of the pathogenic strains of microorganisms and nosocomial infections caused by bacteria and fungi such as Staphylococcus aureus, Escherichia coli, Candida albicans has been observed. The resistance of many of these strains (Pseudomonas aeruginosa, for instance) to popular antibiotics almost reaches the scale of global threat [1]. All these factors motivate the research and development of the novel classes of small molecules suitable as antimicrobials. According to the results of the recent studies of thieno[2,3- $d$ ]pyrimidine derivatives, which showed the antimicrobial activity against both Gram-negative and Gram-positive bacteria [2-5], the molecules bearing this heterocyclic fragment are good candidates for the promising novel class of antibacterials. 
In the last decade, we largely studied the antimicrobial activity of different synthetically prepared thieno[2,3-d]pyrimidines. The highest antimicrobial activity against Gramnegative bacteria (and P. aeruginosa in particular) was determined for 6-heterylthieno[2,3- $d]$ pyrimidines with 1,3,4-oxadiazole I (Figure 1) [6], 2-aminothiazole II [7] and 1,3-benzoxazole moieties attached to thieno[2,3-d]pyrimidin-4-one or 4-thione moieties III [8].<smiles>[R]Sc1ncnc2sc(-c3nnc(-c4ccccc4)o3)c(C)c12</smiles>

I<smiles>[R]Nc1nc(-c2sc3[nH]c(=O)n(-c4ccccc4)c(=O)c3c2C)cs1</smiles>

II<smiles>[R]n1cnc2sc(-c3nc4ccccc4o3)c(C)c2c1=S</smiles>

III<smiles></smiles>

Figure 1. The derivatives of thieno[2,3- $d]$ pyrimidine with antibacterial properties.

The successful application of the hybrid pharmacophore concept achieved by the combination of thieno[2,3- $d$ ]pyrimidine with the other heterocycles has been reported by many other scientific groups. The modification of the position 6 of thieno[2,3- $d]$ pyrimidine with a triazolothiadiazole cycle allowed the obtaining of highly effective antibacterial agents IV, which were also active against $P$. aeruginosa [9]. The antimicrobial and antifungal properties were found to be typical for the compounds $\mathbf{V}$ modified with 1,3,4-oxadiazole [10]; their analogs bore morpholine or piperidine cycles at position 4 of the core heterocycle (VI) also showed antimicrobial properties [11]. Other reports have shown some examples of successful modifications of other positions of the thieno[2,3- $d$ ]pyrimidine system as well as fusion of this ring system with different heterocycles (which led to the effective antimicrobials). The examples tetrahydrobenzo[4,5]thieno[2,3- $d$ ]pyrimidine derivatives [12,13], 5-furyl [14] and 2-thienyl theinopyrimidines fused with triazole [15] or pyrimidine cycles can also be mentioned [16].

The selective action on the specific enzymes of bacterial cells is of the great priority for discovery of the novel mechanisms of antibacterial action. An important advantage of some thieno[2,3- $d$ ]pyrimidine derivatives is their confirmed ability to selectively inhibit the enzymes of different microorganisms such as P. aeruginosa and M. tuberculosis. Some thienopyrimidines were found to be active against isoniazid resistant strains of M. tuberculosis due to their inhibitory activities of enoyl acyl-carrier protein reductase, which do not require activation of catalase-peroxidase $[17,18]$. It was also claimed that 4-amino-thieno[2,3- $d$ ] pyrimidine are the new scaffolds of the selective inhibitors of the QcrB subunit of the electron transport chain enzyme cytochrome bc1, which is a novel target for antitubercular agents [19]. The inhibition of QcrB results in the blockade of oxidative phosphorylation, which leads to the total death of M. tuberculosis cells [20].

One of the newly disclosed mechanisms of antimicrobial action is the inhibition of tRNA (Guanine37- $\mathrm{N}^{1}$ )-methyltransferase (TrmD) [21]. The derivatives of thieno[2,3- $d$ ] pyrimidine of general I (Figure 2) were the first series inhibitors for TrmD isolated from Haemophilus 
influenzae [22]. The most effective of these derivatives were the ligands that deeply immerse into the hydrophobic pocket, attaching to the site of methionine fragment of $S$-adenosylmethionine (SAM) cofactor. As a result of competing processes, the further methylation of tRNA is blocked; in this example of inhibitors, there is a compound II (Figure 2) with the terminal imidazole fragment. Another paper was about the modification of the structure and synthesis of the combinatorial library of the novel selective inhibitors of bacterial TrmD [23]. The complexes of different TrmD isolated from S. aureus, $P$. aeruginosa or $M$. tuberculosis with the different ligands were studied in detail, which allowed for the discovery of tyrosine-flipping mechanism of inhibitory action. The most active compounds reported were III and IV (Figure 2).

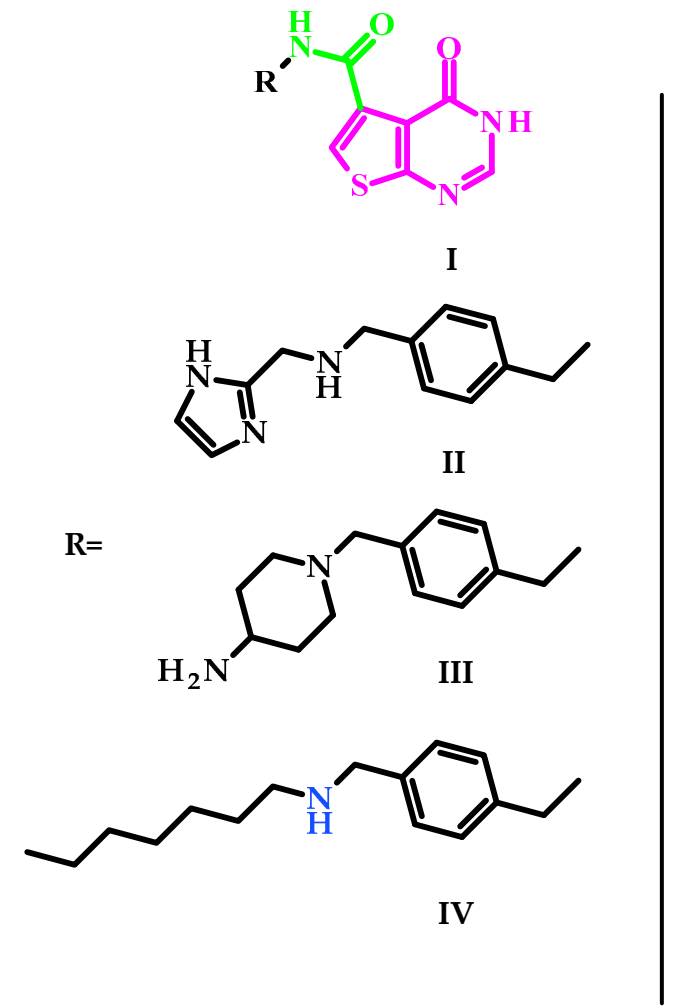

TrmD inhibitors with thienopyrimidinone scaffold<smiles>CCOC(=O)c1ccc2c(c1)nc(-c1ccc(C(F)(F)F)cc1)n2CCN1CCOCC1</smiles><smiles>[R]C(=O)c1ccc2c(c1)nc(C)n2-c1ccc(-c2cccc(NC(=O)c3ccc4nc(C)[nH]c4c3)c2)cn1</smiles>

Antibacterial activity<smiles></smiles>

Figure 2. Design strategy for target compounds with antimicrobial activity.

All the above-mentioned resulted undoubtedly show that modification of thieno[2,3- $d$ ]pyrimidine scaffolds is a promising method for the construction of novel antibacterial agents. Within the framework of the hybrid pharmacophore concept, we decided to modify thienopyrimidine moiety with benzimidazole (Figure 2). Benzimidazole was chosen as the substituent because many of its derivatives were reported as antibacterials [24,25], antituberculosis [26], antifungals [27] and antiparasitics [28] (Figure 2); a high affinity for bacterial enzymes was also reported for this heterocycle. It is also worth mentioning that benzimidazole is among the "privileged sub-structures" for drug discovery [29]. 
The outer membrane of Gram-negative bacteria is a reason for its high non-specific resistance to many antibiotics [30], it is also known that diffusion across the membrane is facilitated for lipophilic molecules. Therefore, the decision to introduce the sulphur atom at position 2 of thienopyrimidine together with the methyl groups at positions 3 and 5 was made in order to increase the lipophilicity.

The aim of this research was to search for effective antibacterials with 6 - $(1 H$-benzimidazol2-yl)-3,5-dimethyl-4-oxo-2-thio-3,4-dihydrothieno[2,3-d]pyrimidine as a key structure.

\section{Materials and Methods}

\subsection{General Information}

All solvents and reagents were acquired from their commercial sources. The melting points were determined in a capillary using an electrothermal IA9100X1 (Bibby Scientific Limited, Staffordshire, UK) digital melting point apparatus. The elemental analyses was performed on a Euro Vector EA-3000 (Eurovector SPA, Redavalle, Italy) microanalyzer and were within $0.4 \%$ of the theoretical values. All the NMR spectra were recorded on Bruker Avance $500{ }^{1} \mathrm{H}$ NMR (500 MHz) and ${ }^{13} \mathrm{C}$ NMR $(125 \mathrm{MHz})$ in DMSO- $d_{6}$. TMS was used as an internal standard. LC-MS analysis of compounds was performed on an Agilent 1100 HPLC apparatus with diode array and mass-selective detectors (Agilent, Santa Clara, CA, USA, LC-MSD SL), Zorbax SB-C18 column $(4.6 \times 15 \mathrm{~mm})$ with chemical ionization at atmospheric pressure (APCI).

\subsection{Molecular Docking Study}

The hardware used is the ASUS VivoBook X530UN S15 with an Intel Core i7 8550U 8thGen and Windows 10, 64-bit operating system. The molecular docking studies were performed with AutoDock Vina and AutoDockTools 1.5.6 programs [31]. The biomolecular target was acquired from Protein Data Bank [32]: TrmD Pseudomonas aeruginosa PDB ID-5ZHN [23]. The construction of the virtual base was done with BIOVIADraw 2017R2; the files were recorder in mol format. The structures were optimized with Chem3D using MM2 algorithm and saved as pdb. Their transformation to pdbqt format was performed with AutoDockTools-1.5.6 [33]. Discovery Studio Visualizer 2017/R2 was used for removal of the molecules of the solvent and the native ligand of the enzyme. The prepared macromolecule was saved in the pdb format. The program AutoDockTools-1.5.6 was used to add polar hydrogen atoms to the stricture of the enzyme and to save it as pdbqt. The size of the Grid box and its center was determined and validated using the native ligand of A subunit: TrmD (PDB ID 5ZHN): $x=40.04, y=107.23, z=-3.40$; size $x=18, y=22, z=20$. To validate the docking method of the reference ligand, compound IV (Figure 2) [23], is extracted and then reused for the redocking process after given charged, set torque and saved as pdbqt. The calculation of the RMSD value was performed with ProFit Results available online. Discovery Studio V17.2.0.16349 was used for the visualization and analysis of the results.

\subsection{Synthesis and Characterization of Compounds}

Ethyl3,5-dimethyl-4-oxo-2-thioxo-1,2,3,4-tetrahydrothieno[2,3-d]pyrimidine-6-carboxylate (which was used as the starting material) was prepared by the method reported in the paper [34].

2.3.1. Synthesis of 3,5-Dimethyl-4-oxo-2-thioxo-1,2,3,4-tetrahydrothieno[2,3- $d$ ]pyrimidine-6carboxylic Acid (2)

To start, $4.20 \mathrm{~g}(0.105 \mathrm{~mol})$ of sodium hydroxide and then $200 \mathrm{~mL}$ of water were added to $10.00 \mathrm{~g}(0.035 \mathrm{~mol})$ of the ester 1 . The reaction mixture was heated to $50-60{ }^{\circ} \mathrm{C}$ and allowed to stir at this temperature for $10 \mathrm{~h}$. Then, the reaction mixture was cooled and acidified with orthophosphoric acid (85\%). The precipitate formed was filtered and washed with plenty of water. The crystalline mass of the product was dried at $60^{\circ} \mathrm{C}$ for 3 days. 
Yield (7.98 g) 89\%, m.p. $>300{ }^{\circ} \mathrm{C}$, grey solid. ${ }^{1} \mathrm{H}$ NMR $\left(500 \mathrm{MHz}, \mathrm{DMSO}-d_{6}\right): \delta 2.70(\mathrm{~s}$, $\left.3 \mathrm{H}, \mathrm{CH}_{3}\right), 3.56\left(\mathrm{~s}, 3 \mathrm{H}, \mathrm{NCH}_{3}\right), 13.56$ (br. s, $\left.1 \mathrm{H}, \mathrm{COOH}\right) .{ }^{13} \mathrm{C} \mathrm{NMR}\left(125 \mathrm{MHz}, \mathrm{DMSO}-d_{6}\right)$ : $\delta 14.6,33.5,117.0,120.1,143.6,152.6,157.5,163.8,175.3$. LC-MS m/z (ES-) $255.0(\mathrm{M}-\mathrm{H})^{-}$. Anal. calcd. for $\mathrm{C}_{9} \mathrm{H}_{8} \mathrm{~N}_{2} \mathrm{O}_{3} \mathrm{~S}_{2}$ (256.30): C, 42.18; H, 3.15; N, 10.93. Found: C, 42.34; H, 3.27; $\mathrm{N}, 11.02$.

2.3.2. Synthesis of 6-(1H-Benzimidazol-2-yl)-3,5-dimethyl-2-thioxo- $1 H$-thieno[2,3- $d$ ]pyrimidin4-one (3)

To start, $3.35 \mathrm{~g}(0.021 \mathrm{~mol})$ of 1,1'-carbonyldiimidazole and $12 \mathrm{~mL}$ of dimethylformamide for peptide synthesis were added to $5.00 \mathrm{~g}(0.0195 \mathrm{~mol})$ of 2 . The reaction mixture was heated at $70-80{ }^{\circ} \mathrm{C}$ for $15 \mathrm{~min}$. Then, $2.22 \mathrm{~g}(0.0205 \mathrm{~mol})$ of $o$-phenylenediamine was added into the reaction mixture, the temperature was raised to $130{ }^{\circ} \mathrm{C}$ and the heating continued for $2.5 \mathrm{~h}$. The reaction mixture was cooled and quenched with water. The precipitate formed was filtered off and washed with plenty of water.

Yield (3.71 g) 58\%, m.p. $>300{ }^{\circ} \mathrm{C}$, yellow solid. ${ }^{1} \mathrm{H}$ NMR (500 MHz, DMSO- $\left.d_{6}\right)$ : $\delta 2.82\left(\mathrm{~s}, 3 \mathrm{H}, \mathrm{CH}_{3}\right), 3.62\left(\mathrm{~s}, 3 \mathrm{H}, \mathrm{NCH}_{3}\right), 7,19(\mathrm{~m}, 2 \mathrm{H}, \mathrm{Ar}-\mathrm{H}), 7,57$ (m, 2H, Ar-H), 12.62 (br. s, $1 \mathrm{H}, \mathrm{NH}) .{ }^{13} \mathrm{C}$ NMR $\left(125 \mathrm{MHz}, \mathrm{DMSO}-d_{6}\right): \delta 14.4,33.0,116.5,119.8,122.2,133.8,145.6$, 151.3, 157.0, 174.2. LC-MS m/z (ES+) $329.0\left(\mathrm{MH}^{+}\right)$. Anal. calcd. for $\mathrm{C}_{15} \mathrm{H}_{12} \mathrm{~N}_{4} \mathrm{OS}_{2}(328.42)$ : C, 54.86; H, 3.68; N, 17.06. Found: C, 54.93; H, 3.77; N, 17.12.

2.3.3. General Method for Synthesis of 2-[6-(1H-Benzimidazol-2-yl)-3,5-dimethyl-4-oxothieno[2,3-d]pyrimidin-2-yl] sulfanyl- $N$-(aryl/heteryl)acetamides (4a-1) and 6- $(1 H-$ benzimidazol-2-yl)-2-(benzylsulfanyl)-3,5-dimethylthieno[2,3- $d$ ]pyrimidin-4(3H)-one (5)

To start, $0.6 \mathrm{mmol}$ of the corresponding chloroacetamide or benzyl chloride and $0.66 \mathrm{mmol}$ of triethylamine were added to the mixture of $0.2 \mathrm{~g}(0.6 \mathrm{mmol})$ of $6-\left(1 H_{-}\right.$ benzimidazol-2-yl)-3,5-dimethyl-2-thioxo-2,3-dihydrothieno[2,3- $d$ ]pyrimidin-4(1H)-one (3) in $4 \mathrm{~mL}$ of dimethylformamide. The reaction mixture was stirred and heating $\left(80^{\circ} \mathrm{C}\right)$ until the clear solution is formed (1-2 h) and then additionally for $2 \mathrm{~h}$. After the cooling reaction, the mixture was diluted with water and the precipitate formed was filtered off and dried. The product was additionally purified via crystallization from ethanol.

2-[6-(1H-Benzimidazol-2-yl)-3,5-dimethyl-4-oxo-thieno[2,3-d]pyrimidin-2-yl]sulfanyl-N -phenyl-acetamide (4a). Yield: $83 \%$, m.p. $>300{ }^{\circ} \mathrm{C}$, yellowish powder. ${ }^{1} \mathrm{H}$ NMR $(500 \mathrm{MHz}$, DMSO- $\left.d_{6}\right): 2.89\left(\mathrm{~s}, 3 \mathrm{H}, \mathrm{CH}_{3}\right), 3.45\left(\mathrm{~s}, 3 \mathrm{H}, \mathrm{NCH}_{3}\right), 4.26\left(\mathrm{~s}, 2 \mathrm{H}, \mathrm{SCH}_{2}\right), 7.07(\mathrm{t}, 1 \mathrm{H}, J=7.4 \mathrm{~Hz}$, Ar-H), 7.22 (m, 2H, Ar-H benzimidazole), 7.32 ( $\mathrm{t}, 2 \mathrm{H}, J=7.4 \mathrm{~Hz}, \mathrm{Ar}-\mathrm{H}), 7.59$ (m, 4H, Ar-H benzimidazole), 10.22 (s, 1H, CONH), 12.41 (br. s, $1 \mathrm{H}, \mathrm{NH}$ benzimidazole). ${ }^{13} \mathrm{C}$ NMR $\left(125 \mathrm{MHz}, \mathrm{DMSO}-d_{6}\right): \delta 15.4,30.5,37.8,119.7,119.9,122.5,124.1,129.3,134.3,139.4,146.5$, 158.3, 159.8, 163.1, 165.8. LC-MS m/z (ES+) $462.0\left(\mathrm{MH}^{+}\right)$. Anal. calcd. for $\mathrm{C}_{23} \mathrm{H}_{19} \mathrm{~N}_{5} \mathrm{O}_{2} \mathrm{~S}_{2}$ (461.57): C, 59.85; H, 4.15; N, 15.17. Found: C, 59.94; H, 4.29; N, 15.23.

2-[6-(1H-benzimidazol-2-yl)-3,5-dimethyl-4-oxo-thieno[2,3-d]pyrimidin-2-yl]sulfanyl-N(p-methyl)acetamide (4b). Yield: 69\%, m.p. $230-231{ }^{\circ} \mathrm{C}$, yellowish powder. ${ }^{1} \mathrm{H}$ NMR $\left(500 \mathrm{MHz}, \mathrm{DMSO}-d_{6}\right): \delta: 2.24\left(\mathrm{~s}, 3 \mathrm{H}, \mathrm{CH}_{3}\right), 2.86\left(\mathrm{~s}, 3 \mathrm{H}, \mathrm{CH}_{3}\right), 3.5\left(\mathrm{~s}, 3 \mathrm{H}, \mathrm{NCH}_{3}\right), 4.23$ $\left(\mathrm{s}, 2 \mathrm{H}, \mathrm{SCH}_{2}\right), 7.12(\mathrm{~d}, 2 \mathrm{H}, J=7.9, \mathrm{Ar}-\mathrm{H}), 7.21(\mathrm{~m}, 2 \mathrm{H}$, Ar-H benzimidazole $), 7.48(\mathrm{~d}$, 2H, J = 7.9, Ar-H), 7.59 (m, 2H, Ar-H benzimidazole), 10.33 (s, 1H, CONH), 12.54 (br. s, 1H, NH benzimidazole). ${ }^{13} \mathrm{C}$ NMR $\left(125 \mathrm{MHz}, \mathrm{DMSO}-d_{6}\right): \delta 15.4,21.0,30.5,37.8,119.6,119.8$, $122.5,129.7,133.0,134.3,136.9,146.5,158.3,159.8,163.1,165.5$. LC-MS m/z (ES+) 476.0 $\left(\mathrm{MH}^{+}\right)$. Anal. calcd. for $\mathrm{C}_{24} \mathrm{H}_{21} \mathrm{~N}_{5} \mathrm{O}_{2} \mathrm{~S}_{2}$ (475.60): C, 60.61; $\mathrm{H}, 4.45 ; \mathrm{N}, 14.73$. Found: $\mathrm{C}, 60.73$; $\mathrm{H}, 4.49 ; \mathrm{N}, 14.86$.

2-\{[6-(1H-Benzimidazol-2-yl)-3,5-dimethyl-4-oxo-3,4-dihydrothieno[2,3-d]pyrimidin-2- yl]thio\}-N(2-ethylphenyl)acetamide (4c). Yield: 65\%, m.p. $291-292{ }^{\circ} \mathrm{C}$, white powder. ${ }^{1} \mathrm{H}$ NMR $\left(500 \mathrm{MHz}, \mathrm{DMSO}-d_{6}\right): \delta: 1.11\left(\mathrm{t}, 3 \mathrm{H}, J=7.4, \mathrm{CH}_{3}\right), 2.61\left(\mathrm{q}, 2 \mathrm{H}, J=7.4, \mathrm{CH}_{2}\right), 2.88(\mathrm{~s}$, $\left.3 \mathrm{H}, \mathrm{CH}_{3}\right), 3.52\left(\mathrm{~s}, 3 \mathrm{H}, \mathrm{NCH}_{3}\right), 4.27\left(\mathrm{~s}, 2 \mathrm{H}, \mathrm{SCH}_{2}\right), 7.15-7.23(\mathrm{~m}, 2 \mathrm{H}$, Ar-H benzimidazole), 7.32 (d, 1H, J = 7.1 Hz, Ar-H), 7.56-7.65 (m, 2H, Ar-H benzimidazole), 9.73 (s, 1H, CONH), 12.56 (br. s, $1 \mathrm{H}, \mathrm{NH}$ benzimidazole). ${ }^{13} \mathrm{C}$ NMR (125 MHz, DMSO- $\left.d_{6}\right): \delta 14.9,15.5,24.3$, $30.5,36.9,119.7,122.5,126.5,126.6,126.6,129.1,134.3,135.9,138.8,146.5,158.3,159.8,163.2$, 
166.2. LC-MS m/z (ES+) $490.2\left(\mathrm{MH}^{+}\right)$. Anal. calcd. for $\mathrm{C}_{25} \mathrm{H}_{23} \mathrm{~N}_{5} \mathrm{O}_{2} \mathrm{~S}_{2}$ (489.62): $\mathrm{C}, 61.33 ; \mathrm{H}$, $4.73 ; \mathrm{N}, 14.30$. Found: C, 61.35; H, 4.80; N, 14.33 .

2-\{[6-(1H-Benzimidazol-2-yl)-3,5-dimethyl-4-oxo-3,4-dihydrothieno[2,3-d]pyrimidin-2-yl]thio\}-N-(4isopropylphenyl)acetamide (4d). Yield: 79\%, m.p. $189-190{ }^{\circ} \mathrm{C}$, yellowish powder. ${ }^{1} \mathrm{H}$ NMR (500 MHz, DMSO-d $\left.d_{6}\right): \delta: 1.16\left(\mathrm{~d}, 6 \mathrm{H}, J=6.5, \mathrm{CH}_{3}\right), 2.86\left(\mathrm{~m}, 4 \mathrm{H}, \mathrm{CH}, \mathrm{CH}_{3}\right)$, 3.49 (s, 3H, CH $\left.\mathrm{CH}_{3}\right), 4.24$ (s, 2H, SCH$), 7.10-7.33$ (m, 4H, Ar-H), 7.41-7.70 (m, 4H, Ar-H), 10.34 (s, 1H, CONH), 12.55 (br. s, $1 \mathrm{H}, \mathrm{NH}$ benzimidazole). ${ }^{13} \mathrm{C}$ NMR (125 MHz, DMSO- $\left.d_{6}\right)$ : $\delta 15.3,24.4,30.4,33.3,37.7,119.5,119.8,122.4,127.0,134.2,137.0,144.1,146.3,158.2,159.7$, 163.0, 165.4, 204.3. LC-MS m/z (ES+) 504.2 ( $\left.\mathrm{MH}^{+}\right)$. Anal. calcd. for $\mathrm{C}_{26} \mathrm{H}_{25} \mathrm{~N}_{5} \mathrm{O}_{2} \mathrm{~S}_{2}(503.65)$ : C, 62.01; H, 5.00; N, 13.91. Found: C, 62.14; H, 5.03; N, 14.06.

2-\{[6-(1H-Benzimidazol-2-yl)-3,5-dimethyl-4-oxo-3,4-dihydrothieno[2,3-d]pyrimidin-2-yl]thio\}-N-[3(trifluoromethyl)phenyllacetamide (4e). Yield: 89\%, m.p. $278-279{ }^{\circ} \mathrm{C}$, yellowish powder. ${ }^{1} \mathrm{H}$ NMR (500 MHz, DMSO- $\left.d_{6}\right): \delta: 2.86\left(\mathrm{~s}, 3 \mathrm{H}, \mathrm{CH}_{3}\right), 3.51\left(\mathrm{~s}, 3 \mathrm{H}, \mathrm{NCH}_{3}\right), 4.28\left(\mathrm{~s}, 2 \mathrm{H}, \mathrm{SCH}_{2}\right), 7.20$ (m, 2H, Ar-H benzimidazole), $7.42(\mathrm{~d}, 1 \mathrm{H}, J=7.4$, Ar-H benzimidazole), 7.53 (d, 1H, J = 7.1, Ar-H), $7.58(\mathrm{t}, 1 \mathrm{H}, \mathrm{J}=7.9$, Ar-H), 7.63 (d, 1H, J = 7.4, Ar-H benzimidazole), $7.77(\mathrm{~d}, 1 \mathrm{H}$, $J=8.2$, Ar-H), 8.11 (s, 1H, Ar-H), 10.78 (s, 1H, CONH), 12.52 (br. s, 1H, NH benzimidazole). ${ }^{13} \mathrm{C}$ NMR $(125 \mathrm{MHz}$, DMSO-d 6 ): $\delta 15.4,19.1,30.5,37.8,56.6,112.2,115.7,119.1,119.6,120.4$, 122.5, 122.6, 123.2, 123.3, 130.7, 134.3, 135.4, 140.2, 143.7, 146.4, 158.2, 159.6, 163.0, 166.5 . LC-MS m/z (ES+) $530.0\left(\mathrm{MH}^{+}\right)$. Anal. calcd. for $\mathrm{C}_{24} \mathrm{H}_{18} \mathrm{~F}_{3} \mathrm{~N}_{5} \mathrm{O}_{2} \mathrm{~S}_{2}$ (529.57): C, 54.43; $\mathrm{H}$, 3.43; N, 13.22. Found: C, 54.58; H, 4.10; N, 13.56 .

2-\{[6-(1H-Benzimidazol-2-yl)-3,5-dimethyl-4-oxo-3,4-dihydrothieno[2,3-d]pyrimidin-2-yl]thio\}-N-(2ethoxyphenyl)acetamide (4f). Yield: 85\%, m.p. $240-241^{\circ} \mathrm{C}$, grey powder. ${ }^{1} \mathrm{H}$ NMR $(500 \mathrm{MHz}$, DMSO- $\left.d_{6}\right): \delta: 1.31\left(\mathrm{t}, 3 \mathrm{H}, J=6.8, \mathrm{CH}_{3}\right), 2.89\left(\mathrm{~s}, 3 \mathrm{H}, \mathrm{CH}_{3}\right), 3.55\left(\mathrm{~s}, 3 \mathrm{H}, \mathrm{NCH}_{3}\right), 4.1(\mathrm{q}$, $\left.2 \mathrm{H}, \mathrm{J}=6.8, \mathrm{CH}_{2}\right), 4.31\left(\mathrm{~s}, 2 \mathrm{H}, \mathrm{SCH}_{2}\right), 6.9(\mathrm{t}, 2 \mathrm{H}, \mathrm{J}=6.8, \mathrm{Ar}-\mathrm{H}), 7.01-7.06(\mathrm{~m}, 2 \mathrm{H}, \mathrm{Ar}-\mathrm{H}), 7.22$ (m, 2H, Ar-H benzimidazole), 7.55-7.64 (m, 2H, Ar-H benzimidazole), 7.94 (m, 1H, Ar-H), 9.3 (s, 1H, CONH), 12.44 (br. s, $1 \mathrm{H}, \mathrm{NH}$ benzimidazole). ${ }^{13} \mathrm{C}$ NMR (125 MHz, DMSO- $\left.d_{6}\right): \delta$ 15.0, 15.4, 30.6, 37.4, 64.5, 112.2, 112.9, 119.2, 119.7, 120.8, 122.2, 122.6, 123.4, 125.2, 127.7, $134.3,135.5,143.8,146.4,149.2,158.3,159.6,163.1,165.9$. LC-MS m/z (ES+) $506.0\left(\mathrm{MH}^{+}\right)$. Anal. calcd. for $\mathrm{C}_{25} \mathrm{H}_{23} \mathrm{~N}_{5} \mathrm{O}_{3} \mathrm{~S}_{2}$ (505.62): C, 59.39; $\mathrm{H}, 4.59 ; \mathrm{N}, 13.85$. Found: $\mathrm{C}, 59.47 ; \mathrm{H}, 4.62$; $\mathrm{N}, 13.90$.

2-\{[6-(1H-Benzimidazol-2-yl)-3,5-dimethyl-4-oxo-3,4-dihydrothieno[2,3-d]pyrimidin-2-yl]thio\}-N-(4ethoxyphenyl)acetamide (4g). Yield: $92 \%$, m.p. $175-176{ }^{\circ} \mathrm{C}$, white powder. ${ }^{1} \mathrm{H} \mathrm{NMR}(500 \mathrm{MHz}$, DMSO-d $\left.d_{6}\right): \delta: 1.29\left(\mathrm{t}, 3 \mathrm{H}, J=6.8, \mathrm{CH}_{3}\right), 2.86\left(\mathrm{~s}, 3 \mathrm{H}, \mathrm{CH}_{3}\right), 3.5\left(\mathrm{~s}, 3 \mathrm{H}, \mathrm{NCH}_{3}\right), 3.97(\mathrm{~m}, 2 \mathrm{H}$, $\left.\mathrm{CH}_{2}\right), 4.21$ (s, 2H, SCH 2$), 6.87(\mathrm{~d}, 2 \mathrm{H}, \mathrm{J}=7.7, \mathrm{Ar}-\mathrm{H}), 7.21$ (m, 2H, Ar-H benzimidazole), $7.48(\mathrm{~d}, 2 \mathrm{H}, J=7.7, \mathrm{Ar}-\mathrm{H}), 7.53-7.63$ (m, 2H, Ar-H benzimidazole), 10.26 (s, 1H, CONH), 12.54 (br. s, $1 \mathrm{H}, \mathrm{NH}$ benzimidazole). ${ }^{13} \mathrm{C}$ NMR $\left(125 \mathrm{MHz}, \mathrm{DMSO}-d_{6}\right): \delta 15.1,15.3,30.4,37.6$, $63.5,114.9,119.5,121.2,122.4,132.3,134.2,146.3,155.1,158.2,159.7,163.0,165.1$. LC-MS $\mathrm{m} / \mathrm{z}(\mathrm{ES}+) 506.0\left(\mathrm{MH}^{+}\right)$. Anal. calcd. for $\mathrm{C}_{25} \mathrm{H}_{23} \mathrm{~N}_{5} \mathrm{O}_{3} \mathrm{~S}_{2}$ (505.62): C, 59.39; $\mathrm{H}, 4.59 ; \mathrm{N}, 13.85$. Found: $\mathrm{C}, 59.41 ; \mathrm{H}, 4.66 ; \mathrm{N}, 13.94$.

2-\{[6-(1H-Benzimidazol-2-yl)-3,5-dimethyl-4-oxo-3,4-dihydrothieno[2,3-d]pyrimidin-2-yl]thio\}-N-(3,4dimethoxyphenyl)acetamide (4h). Yield: $67 \%$, m.p. $185-186{ }^{\circ} \mathrm{C}$, white powder. ${ }^{1} \mathrm{H}$ NMR $(500$ $\left.\mathrm{MHz}, \mathrm{DMSO}-d_{6}\right): \delta: 2.86\left(\mathrm{~s}, 3 \mathrm{H}, \mathrm{CH}_{3}\right), 3.50\left(\mathrm{~s}, 3 \mathrm{H}, \mathrm{NCH}_{3}\right), 3.71\left(\mathrm{~m}, 6 \mathrm{H}, \mathrm{OCH}_{3}\right), 4.21(\mathrm{~s}$, $\left.2 \mathrm{H}, \mathrm{SCH}_{2}\right), 6.88-6.91(\mathrm{~d}, 1 \mathrm{H}, J=8.5, \mathrm{Ar}-\mathrm{H}), 7.06-7.09(\mathrm{~d}, 1 \mathrm{H}, J=8.5, \mathrm{Ar}-\mathrm{H}), 7.21(\mathrm{~m}, 2 \mathrm{H}$, Ar-H benzimidazole), 7.31 (m, 1H, Ar-H), 7.54-7.63 (m, 2H, Ar-H benzimidazole), 10.28 (s, 1H, CONH), 12.53 (br. s, $1 \mathrm{H}, \mathrm{NH}$ benzimidazole). ${ }^{13} \mathrm{C}$ NMR (125 MHz, DMSO- $\left.d_{6}\right): \delta$ 15.3, 30.4, 37.7, 55.8, 56.1, 104.8, 111.6, 112.0, 112.5, 119.0, 119.5, 122.4, 123.2, 132.9, 134.1, $145.5,146.3,149.0,158.2,159.7,163.0,165.2$. LC-MS m/z (ES+) $522.0\left(\mathrm{MH}^{+}\right)$. Anal. calcd. for $\mathrm{C}_{25} \mathrm{H}_{23} \mathrm{~N}_{5} \mathrm{O}_{4} \mathrm{~S}_{2}$ (521.62): C, 57.57; $\mathrm{H}, 4.44 ; \mathrm{N}, 13.43$. Found: $\mathrm{C}, 57.63 ; \mathrm{H}, 4.52 ; \mathrm{N}, 13.48$.

2-\{[6-(1H-Benzimidazol-2-yl)-3,5-dimethyl-4-oxo-3,4-dihydrothieno[2,3-d]pyrimidin-2-yl]thio\}-N-(5chloro-2-methylphenyl)acetamide (4i). Yield: $86 \%$, m.p. $>300{ }^{\circ} \mathrm{C}$, yellowish powder. ${ }^{1} \mathrm{H}$ NMR (500 MHz, DMSO-d ${ }_{6}$ ): $\delta: 2.25$ (s, 3H, $\left.\mathrm{CH}_{3}\right), 2.87\left(\mathrm{~s}, 3 \mathrm{H}, \mathrm{CH}_{3}\right), 3.50(\mathrm{~s}, 3 \mathrm{H}$, $\left.\mathrm{NCH}_{3}\right), 4.29\left(\mathrm{~s}, 2 \mathrm{H}, \mathrm{SCH}_{2}\right), 7.12-7.14(\mathrm{~m}, 1 \mathrm{H}, \mathrm{Ar}-\mathrm{H}), 7.18-7.26$ (m, 3H, Ar-H benzimidazole), 7.50-7.49 (m, 3H, Ar-H benzimidazole), 9.83 (s, 1H, CONH), 12.54 (br. s, 1H, NH 
benzimidazole). ${ }^{13} \mathrm{C}$ NMR (125 MHz, DMSO- $\left.d_{6}\right): \delta 15.4,17.9,30.4,37.0,119.6,122.4,124.4$, $125.4,130.3$, 130.7, 132.3, 134.2, 137.8, 146.3, 158.2, 159.7, 163.0, 166.2. LC-MS m/z (ES+) $510.0\left(\mathrm{MH}^{+}\right)$. Anal. calcd. for $\mathrm{C}_{24} \mathrm{H}_{20} \mathrm{ClN}_{5} \mathrm{O}_{2} \mathrm{~S}_{2}$ (510.04): C, 56.52; H, 3.95; N, 13.73. Found: C, 56.51; H, 3.99; N, 13.83 .

2-\{[6-(1H-Benzimidazol-2-yl)-3,5-dimethyl-4-oxo-3,4-dihydrothieno[2,3-d]pyrimidin-2-yl]thio\}-N-(3chloro-4-fluorophenyl)acetamide $(4 j)$. Yield: 77\%, m.p. $214-215{ }^{\circ} \mathrm{C}$, white powder. ${ }^{1} \mathrm{H}$ NMR (500 MHz, DMSO-d $)$ ) $\delta: 2.87\left(\mathrm{~s}, 3 \mathrm{H}, \mathrm{CH}_{3}\right), 3.52\left(\mathrm{~s}, 3 \mathrm{H}, \mathrm{NCH}_{3}\right), 4.24(\mathrm{~s}, 2 \mathrm{H}$, $\left.\mathrm{SCH}_{2}\right), 7.20$ (m, 2H, Ar-H benzimidazole), $7.37(\mathrm{t}, 1 \mathrm{H}, J=9.0, \mathrm{Ar}-\mathrm{H}), 7.49(\mathrm{~m}, 1 \mathrm{H}, \mathrm{Ar}-\mathrm{H})$, 7.58 (m, 2H, Ar-H benzimidazole), 7.88 (d, 1H, Ar-H), 10.54 (s, 1H, CONH), 12.46 (br. s, $1 \mathrm{H}, \mathrm{NH}$ benzimidazole). ${ }^{13} \mathrm{C}$ NMR $\left(125 \mathrm{MHz}, \mathrm{DMSO}-d_{6}\right): \delta 15.3,30.4,37.6,117.4,117.6$, $119.5,119.9,119.9,121.0,122.4,134.2,136.5,146.3,158.1,159.5,162.9,166.1$. LC-MS m/z (ES+) $514.0\left(\mathrm{MH}^{+}\right)$. Anal. calcd. for $\mathrm{C}_{23} \mathrm{H}_{17} \mathrm{ClFN}_{5} \mathrm{O}_{2} \mathrm{~S}_{2}$ (514.00): C, 53.75; H, 3.33; N, 13.63. Found: C, 53.76; H, 3.48; N, 13.66 .

Ethyl 4-[(\{[6-(1H-Benzimidazol-2-yl)-3,5-dimethyl-4-oxo-3,4-dihydrothieno[2,3-d]pyrimidin-2yllthiolacetyl)aminolbenzoate (4k). Yield: 78\%, m.p. $208-209{ }^{\circ} \mathrm{C}$, yellowish powder. ${ }^{1} \mathrm{H}$ NMR (500 MHz, DMSO- $\left.d_{6}\right): \delta: 1.31\left(\mathrm{t}, 3 \mathrm{H}, J=6.3, \mathrm{CH}_{3}\right), 2.88\left(\mathrm{~s}, 3 \mathrm{H}, \mathrm{CH}_{3}\right), 3.54(\mathrm{~s}$, $\left.3 \mathrm{H}, \mathrm{NCH}_{3}\right), 4.29$ (s, 2H, SCH$), 7.2$ (m, 2H, Ar-H benzimidazole), 7.59 (m, 2H, Ar-H benzimidazole), 7.73 (d, 2H, J = 8.0, Ar-H), 7.93 (d, 2H, J = 8.0, Ar-H), 10.56 (s, 1H, CONH), 12.37 (br. s, $1 \mathrm{H}, \mathrm{NH}$ benzimidazole). ${ }^{13} \mathrm{C}$ NMR (125 MHz, DMSO- $\left.d_{6}\right): \delta 14.7,15.4,30.5$, 37.9, 61.0, 119.1, 119.6, 122.5, 125.1, 130.8, 134.3, 143.7, 146.4, 158.2, 159.6, 163.0, 165.8, 166.5 LC-MS m/z (ES+) $534.0\left(\mathrm{MH}^{+}\right)$. Anal. calcd. for $\mathrm{C}_{26} \mathrm{H}_{23} \mathrm{~N}_{5} \mathrm{O}_{4} \mathrm{~S}_{2}$ (533.63): C, 58.52; $\mathrm{H}, 4.34$; $\mathrm{N}, 13.12$. Found: $\mathrm{C}, 58.61 ; \mathrm{H}, 4.36 ; \mathrm{N}, 13.16$.

2-\{[6-(1H-Benzimidazol-2-yl)-3,5-dimethyl-4-oxo-3,4-dihydrothieno[2,3-d]pyrimidin-2-yl]thio\}-N-1,3thiazol-2-ylacetamide (4l). Yield: $83 \%$, m.p. > $300{ }^{\circ} \mathrm{C}$, yellowish powder. ${ }^{1} \mathrm{H} \mathrm{NMR}(500 \mathrm{MHz}$, DMSO- $\left.d_{6}\right): \delta: 2.85\left(\mathrm{~s}, 3 \mathrm{H}, \mathrm{CH}_{3}\right), 3.50\left(\mathrm{~s}, 3 \mathrm{H}, \mathrm{NCH}_{3}\right), 4.34\left(\mathrm{~s}, 2 \mathrm{H}, \mathrm{SCH}_{2}\right), 7.04-7.30(\mathrm{~m}, 3 \mathrm{H}, \mathrm{Ar}-$ $\mathrm{H}), \quad 7.42-7.70(\mathrm{~m}, 3 \mathrm{H}, \mathrm{Ar}-\mathrm{H}), 12.51(\mathrm{~s}, 2 \mathrm{H}, \mathrm{CONH}$ and $\mathrm{NH}$ benzimidazole $)$. ${ }^{13} \mathrm{C}$ NMR $\left(125 \mathrm{MHz}\right.$, DMSO- $\left.d_{6}\right)$ : $\delta 15.4,30.5,36.3,114.2,119.6,122.5,134.3,138.3,146.4$, 158.2, 158.5, 159.3, 162.9, 166.4. LC-MS m/z (ES+) $469.0\left(\mathrm{MH}^{+}\right)$. Anal. calcd. for $\mathrm{C}_{20} \mathrm{H}_{16} \mathrm{~N}_{6} \mathrm{O}_{2} \mathrm{~S}_{3}(468,58):$ C, 51.27; H, 3.44; N, 17.94. Found: C, 51.28; H, 3.47; N, 17.97.

6-(1H-benzimidazol-2-yl)-2-(benzylsulfanyl)-3,5-dimethylthieno[2,3-d]pyrimidin-4(3H)-one (5). Yield: $72 \%$, m.p. $146-147^{\circ} \mathrm{C}$, pale yellow powder. ${ }^{1} \mathrm{H}$ NMR $\left(500 \mathrm{MHz}, \mathrm{DMSO}-d_{6}\right): \delta 2.88$ (s, $\left.3 \mathrm{H}, \mathrm{CH}_{3}\right), 3.45\left(\mathrm{~s}, 3 \mathrm{H}, \mathrm{NCH}_{3}\right), 4.53\left(\mathrm{~s}, 3 \mathrm{H}, \mathrm{SCH}_{2}\right), 7.22(\mathrm{~m}, 2 \mathrm{H}$, Ar-H benzimidazole $), 7.28(\mathrm{t}$, $1 \mathrm{H}, J=7.1 \mathrm{~Hz}, \mathrm{Ar}-\mathrm{H}), 7.34(\mathrm{t}, 2 \mathrm{H}, J=7.4 \mathrm{~Hz}, \mathrm{Ar}-\mathrm{H}), 7.49$ (d, 2H, J = 7.1 Hz, Ar-H), 7.52-7.68 (m, 2H, Ar-H benzimidazole), 12.50 (br. s, $1 \mathrm{H}, \mathrm{NH}$ benzimidazole). ${ }^{13} \mathrm{C}$ NMR (125 MHz, DMSO- $\left.d_{6}\right): \delta 15.4,30.4,36.5,112.2,119.2 ; 119.7,122.4,122.63,123.4,128.1,129.1,129.9,134.4$, $135.5,136.6,143.8,146.5,158.4,159.7,163.2$. LC-MS m/z (ES+) 419.0 $\left(\mathrm{MH}^{+}\right)$. Anal. calcd. for $\mathrm{C}_{22} \mathrm{H}_{18} \mathrm{~N}_{4} \mathrm{OS}_{2}$ (418.54): C, 63.13; $\mathrm{H}, 4.33 ; \mathrm{N}, 13.39$. Found: $\mathrm{C}, 63.25 ; \mathrm{H}, 4.37 ; \mathrm{N}, 13.39$.

\subsection{Antimicrobial Activity Study}

According to the international recommendations and national standards [35-37], the following strains of micro-organisms were used as the test strains Staphylococcus aureus ATCC 25923, Escherichia coli ATCC 25922, Pseudomonas aeruginosa ATCC 27853, Proteus vulgaris ATCC 4636, Bacillus subtilis ATCC 6633 and Candida albicans ATCC653/885.

The microbial suspension of microorganisms was prepared using a Densi-La-Meter device (manufactured by PLIVA-Lachema, Czech Republic; wavelength $540 \mathrm{~nm}$ ). The microbial load was $10^{7}$ microbial cells per $1 \mathrm{~mL}$ of medium and was established according to the MacFarland standard. An 18-24-h microorganism culture was used for the test. Muller-Hinton («HIMedia Laboratorles Pvt. Ltd. India») and Sabouraud agars («HIMedia Laboratorles Pvt. Ltd. India») were used in the studies. The studied compounds were administered in the form of DMSO solutions (concentration $100 \mu \mathrm{g} / \mathrm{mL}$ ) in $0.3-\mathrm{mL}$ aliquots, and Streptomycin was used in the form of a solution in DMSO $(100 \mu \mathrm{g} / \mathrm{mL})$. The measurement for each sample was repeated three times. The antibacterial activity was assessed by measuring the growth inhibition zones of the corresponding microorganism. 


\section{Results and Discussion}

\subsection{Docking Study}

The discovery of the mechanism of selective inhibition of TrmD from different bacteria, which was confirmed for some carboxamide derivatives of thienopyrimidinones [23], allows for the estimation of the binding affinity of the thenopyrimidine structural analogs to TrmD with the help of molecular docking instruments. The molecular docking of the constructed hybrids was performed to the active site of selective inhibitors of TrmD; the model of the enzyme isolated from P. aeruginosa was used for the studies. Macromolecule of TrmD is a homodimer with two identical active sites for binding of its cofactor SAM. Among the TrmD inhibitors, N-(4-((octylamino)methyl)benzyl)-4-oxo-3, 4-dihydrothieno[2,3-d]pyrimidine-5-carboxamide IV is the only known compound able to interact with both binding sites of SAM. It is a competitive inhibitor of TrmD with antibacterial activity against Gram-negative and Gram-positive bacteria and even Mycobacteria tuberculosis. The compound IV we used as the reference ligand for the assessment affinity of the novel derivatives of 6-(1H-benzimidazol-2-yl)-3,5-dimethyl-2-thio-2,3-dihydrothieno[2,3- $d$ ] pyrimidin-4(1H)-one. After redocking the binding energy of the reference ligand was $-8.2 \mathrm{kcal} / \mathrm{mol}$. The capacity of the docking algorithm for experimental modeling is presented in Figure $3 \mathrm{a}$ and in the Table 1 . The validity of the docking study can be estimated by the value of RMSD, which characterizes the degree of reliable docking probability. For our research the RSMD value between the experimental and the reference conformation of the compound IV equals $1.952 \AA$; therefore, the study is reliable [38].

The reference interaction neatly reproduces the spatial arrangement of the ligand (yellow structure) comparatively to its natural position (blue structure) (Figure 3a). The ligand conformation is stabilized by the three loops of the active site, which are cover, bottom and wall loops [22,23]; all those crucial for ligand activity interactions (such as interaction with the tyrosine residues (Tyr141 Ta 120)) were also well modelled.

The target thieno[2,3- $d$ ]pyrimidines showed much lesser values of the binding energies $(-9.5$ to $-10.8 \mathrm{kcal} / \mathrm{mol})$ comparatively to the native ligand $(-8.2 \mathrm{kcal} / \mathrm{mol})$. The only exception was the derivative 3 , the scoring function value of which was $-7.9 \mathrm{kcal} / \mathrm{mol}$. The less favorable value of the scoring function in this case is explained by possible stabilization of the ligand conformation at the entrance to the hydrophobic pocket without the interaction with the amino acids of the SAM active site cavity. The visualization of the arrangements of the 4a-1 molecules demonstrates their similarity to the native ligand (Figure 3); the character and the number of hydrophobic interactions allow for stabilization of the durable conformation in the active site of the enzyme (Table 1).

More detailed analysis of the arrangements of the ligands 4a-1 shows the possibility of their longitudinal and deep immersion into the hydrophobic pocket with the ability to block the SAM methionine binding fragment, which includes interaction with the residues of glutamate (Gln95) and glycine (Gly 118, 145, 146) (Table 1, Figure 3b,c). The possibility of all the important hydrophobic interactions with the amino acids of the cover loop (proline (Pro94), the bottom loop (leucine (Leu143), tyrosine (Tyr143), isoleucine (Ile138)) and the wall loop (tyrosine (Tyr120) and glycine (Gly121)) was predicted. For all of the ligands, the ability of thieno[2,3- $d$ ]pyrimidine core structure to engage in hydrophobic interactions with the isopropyl substituent of valine (Val142), which according to the experimental results does not contribute into attachment of the ligand, although it was visualized in close proximity to the amino acids of the active site, proline (Pro94) in particular.

According to the docking studies results for all the thienopyrimidines 4 and 5 the high affinity level to the inhibitor binding site of TrmD isolated from P. aeruginosa was determined. The best affinity was found for the compound $\mathbf{4 d}$ with an iso-propyl substituent at the arylacetamide fragment (Figure $3 b, c)$. 


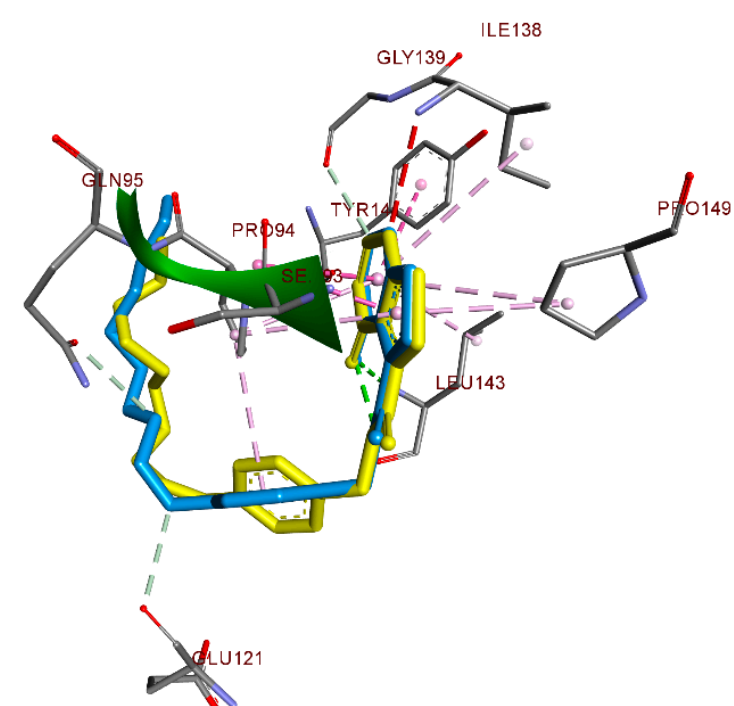

(a)

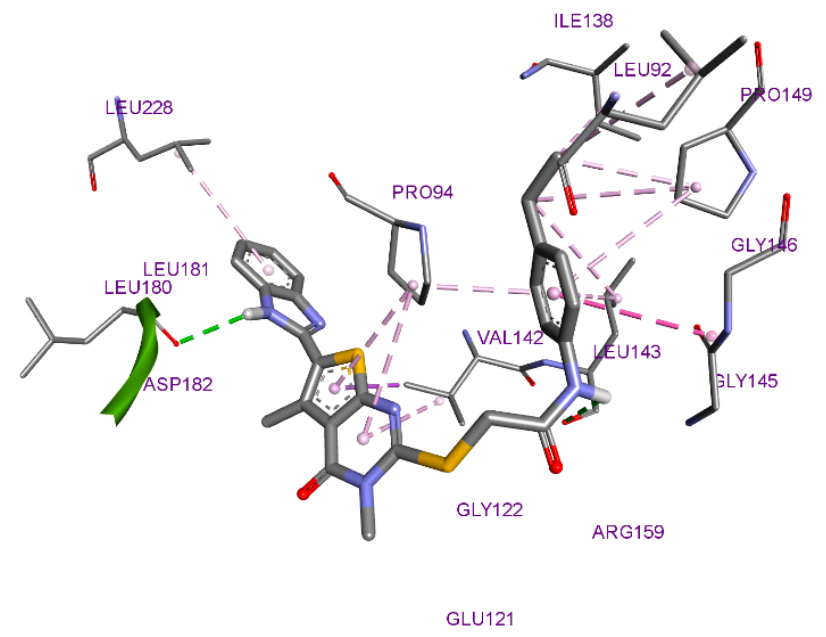

(b)

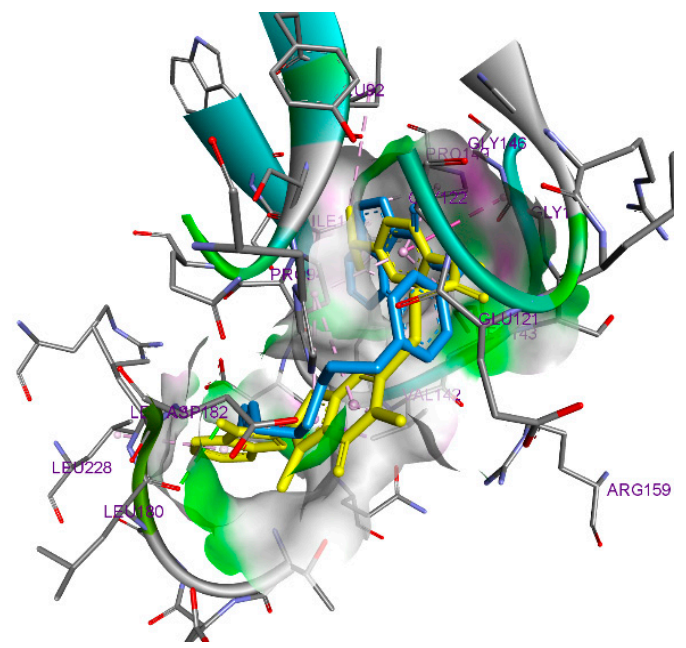

(c)

Figure 3. 3D visualization: (a) the arrangement of the reference native ligand (blue structure) [23] and the reference ligand (yellow structure) in the active site of TrmD of P. aeruginosa; (b) interaction of $4 \mathbf{d}$ with amino acids of the active site of TrmD inhibitors; (c) conformations of both the native inhibitor (blue structure) and the ligand $\mathbf{4 d}$.

\subsection{Chemical Synthesis}

To achieve the objective of constructing of novel derivatives combining thieno[2,3d]pyrimidine and benzimidazole fragments, the synthesis of the starting 3,5-dimethyl4-oxo-2-thioxo- $1 H$-thieno[2,3- $d$ ]pyrimidine-6-carboxylic acid 2 by hydrolysis of the previously reported and readily available ethyl ester 1 (Scheme 1) [34] was carried out. In particular, the high solubility of the starting ester 1 in alkaline water media allows for its hydrolysis at slight heating $\left(50-60^{\circ} \mathrm{C}\right)$ during a 10 hour period. These were found to be the optimal reaction conditions because the rise in the reaction temperature or the increase of alkali concentration produces more admixtures. The attempts to increase the solubility of the starting ester by addition of organic solvents such as ethanol or 1,4-dioxane decrease the yield of the acid 2 due to the problems with the product 3 precipitation. 
Table 1. The results of the target thieno[2,3- $d$ ]pyrimidines $3,4 a-1,5$ and the reference ligand docking studies to the active site of $P$. aeruginosa TrmD.

\begin{tabular}{|c|c|c|c|c|}
\hline Ligand & $\begin{array}{l}\text { Binding Energy } \\
\text { Kcal/Mol }\end{array}$ & Hydrophobic Interaction & Hydrogen Interaction & Other Interaction \\
\hline Reference ligand & -8.2 & $\begin{array}{l}\text { Tyr141, Ser93(2) \#, Pro94 (4), Pro149(2), Ile138, } \\
\text { Leu143, Gly 45, Gly146 }\end{array}$ & $\begin{array}{l}\text { Leu143, Gln95, Glu121, } \\
\text { Gly139, Asp182 }\end{array}$ & - \\
\hline 3 & -7.9 & \multirow{2}{*}{$\begin{array}{l}\text { Val142(3)*, Glu121, Gly122*, Pro94(3) } \\
\text { Glu121(2), Gly122(2)*,Gly145, Gly146, } \\
\text { Val142(3)*, Pro94(3), Leu143, Pro149 }\end{array}$} & Arg159, Leu143 & - \\
\hline $4 a$ & -9.3 & & Tyt120, Thr177* & Asp182 (Pi-Anion) \\
\hline $4 b$ & -10.8 & \multirow{2}{*}{$\begin{array}{l}\text { Val142(2)*, Gly145, Gly146, Ile138, Leu143(2), } \\
\text { Tyr141, Pro94, Pro149 } \\
\text { Glu121(2), Gly122(2)*, Gly145, Gly146, Pro94(3), } \\
\text { Ile138, Leu143(2), Tyr141, Val142 *(2), Pro149 }\end{array}$} & $\begin{array}{l}\text { Arg159*, Gly146, Tyr120, } \\
\text { Ser144*, Thr177(2)* }\end{array}$ & Asp159*(Pi-Anion $)$ \\
\hline $4 c$ & -10.6 & & Gln95, Leu92*, Tyr91* & Asp159* (Pi-Cation) \\
\hline $4 d$ & -10.9 & $\begin{array}{l}\text { Gly145, Gly146, Leu92* } \\
\text { Ile138, Pro149(2), Leu143(3), Ile138, } \\
\text { Leu228*, Pro94(3), Val142 *, Pro149 }\end{array}$ & Leu180*, Leu143 & 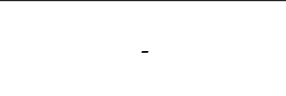 \\
\hline $4 e$ & -10.6 & $\begin{array}{l}\text { Val142(2) *, Gly179, Leu180*, Ser144, Gly145(2), } \\
\text { Gly146, Pro94(3), Pro149, Leu228 }\end{array}$ & Leu180*, Leu143, Thr177* & $\begin{array}{l}\text { Leu92* } \\
\text { Gly145*, Ser93, (Halogen } \\
\text { Fluorine) }\end{array}$ \\
\hline $4 f$ & -10.0 & $\begin{array}{l}\text { Val142*, Gly145(2), Gly146, Leu228 *, Pro94(3), } \\
\text { Leu143, Pro149 }\end{array}$ & Leu180*, Leu143, Leu92* & Asp182 \\
\hline $4 \mathrm{~g}$ & -9.9 & $\begin{array}{l}\text { Val142(2)*, Gly145(2), Gly146, } \\
\text { Leu228 *, Pro94(3), Leu143, Pro149 }\end{array}$ & Leu180*, Leu143, Leu92* & - \\
\hline $4 h$ & -9.8 & \multirow{2}{*}{$\begin{array}{l}\text { SER93, Pro94(4), Glu121, Gly122*, Val142(2) } \\
\text { *,Leu143, Pro149 } \\
\text { Val142(2)*, Gly145(2), Gly146, Pro94(4), } \\
\text { Leu143(2), Leu228 *, Pro149 }\end{array}$} & $\begin{array}{l}\text { Gln95, Leu143, Gly139*, } \\
\text { Trp136 }\end{array}$ & $\operatorname{Arg} 159(2) *$ \\
\hline $4 \mathbf{i}$ & -10.4 & & Leu180*, Leu143, & - \\
\hline $4 \mathbf{j}$ & -10.3 & $\begin{array}{l}\text { Val142(2)*, Gly145, Gly146, Pro94(4), Leu143(2), } \\
\text { Leu228*, Pro149 }\end{array}$ & Leu180*, Leu143, & Ser93 (Halogen Fluorine) \\
\hline $4 k$ & -10.1 & $\begin{array}{l}\text { Val142(3) *, Gly179*, Leu180*, Gly145, Gly146, } \\
\text { Leu228*, Pro94(3), Pro149 }\end{array}$ & Leu143, Glu121, Ser137* & Asp182 (Pi-Anion) \\
\hline 41 & -9.5 & Val142(2) *, Gly145, Gly146, Pro94(2), Leu228* & Leu143, Thr177* & Asp182 (Pi-Anion) \\
\hline 5 & -9.5 & $\begin{array}{l}\text { Glu121(2), Gly122(2) *, Gly145, Gly146, Val142(2) } \\
\text { *, Pro94(3), Leu143, Pro149 }\end{array}$ & Tyr120, Ser144*, Thr177* & Asp182 (Pi-Anion) \\
\hline
\end{tabular}

\# The number of bonds is given in brackets. ${ }^{*}$ The amino acids that do not interact with native ligand in the experiment.

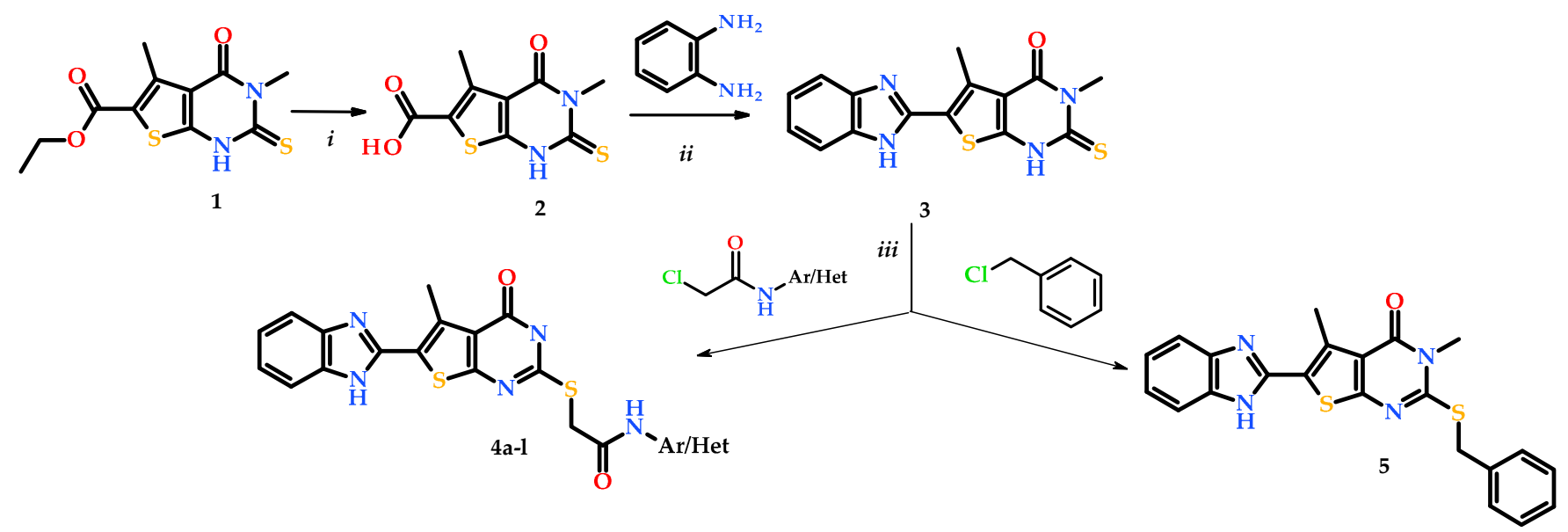

Scheme 1. Synthesis of target thieno[2,3-d]pyrimidine derivatives 4a-1 and 5. Reagent and conditions: (i) 1.1 ( 0.035 mol), $\mathrm{NaOH}(0.105 \mathrm{~mol}), \mathrm{H}_{2} \mathrm{O}, 200 \mathrm{~mL} ; 2 . \mathrm{H}_{3} \mathrm{PO}_{4}(0.110 \mathrm{~mol})$; (ii) 1.2 (0.0195 mol), CDI (0.021 mol) DMF, 70-80 ${ }^{\circ} \mathrm{C}$; 2. o-phenylenediamine $(0.0205 \mathrm{~mol}), \mathrm{DMF}, 130^{\circ} \mathrm{C}$; (iii) $3(0.6 \mathrm{mmol})$, alkylating agent $(0.6 \mathrm{mmol}), \mathrm{NEt}_{3}(0.66 \mathrm{mmol}), \mathrm{DMF}$ $(4 \mathrm{~mL}), 80^{\circ} \mathrm{C}, 3.5 \mathrm{~h}$.

The further synthesis of compound 3 combining thieno[2,3- $d$ ]pyrimidine and benzimidazole fragments was carried out via interaction of 5-methyl-4-oxo-2-thioxo-1,2,3,4tetrahydrothieno[2,3- $-d]$ pyrimidine-6-carboxylic acid 2 imidazolide with $o$-phenylediamine in the way similarly to that reported previously [39]. In this case, the high solubility of the intermediate imidazolide of the acid $\mathbf{2}$ in such a highly-boiling solvent suitable for peptide synthesis as DMF allows for acylation and heterocyclization as a one pot procedure. It is a clear advantage that formation of benzimidazole ring takes place without any addi- 
tional catalysts needed. The isolation of the product 3 is also an advantageous stage as it requires only dilution of the reaction mixture with water and further filtration of the crystalline product.

In the ${ }^{1} \mathrm{H}$ NMR spectrum of the compound 3 the signal of thiophene methyl group at $2.82 \mathrm{ppm}$ and the signal of methyl group at position 3 of thieno[2,3-d]pyrimidine at $3.62 \mathrm{ppm}$ are observed as singlets. The benzimidazole part of the molecule produces two multiplets at 7.19 and $7.57 \mathrm{ppm}$. The signal of NH proton of benzimidazole ring is observed at $12.62 \mathrm{ppm}$, while the signal of $\mathrm{NHC}=\mathrm{S}$ is not observed because of the deuterium exchange.

Acetamide and benzyl fragments were introduced into the hybrid molecule 3 by alkylation of the sulfur atom at position 2 of thieno[2,3-d]pyrimidine system. The reaction was carried out in DMF using trimethylamine as a catalyst. The compound 3 is rather poorly soluble in DMF and therefore its alkylation requires relatively high temperature and prolonged time. The reaction is possible due to the good solubility of the products 4 and 5 in hot DMF.

The structure of the obtained compounds $4 a-1$ and 5 was assigned using the data of ${ }^{1} \mathrm{H}$ and ${ }^{13} \mathrm{C}$ NMR as well as LC-MS. If compared with the starting compound 3 the signals of methyl substituents of thienopyrimidine ring in ${ }^{1} \mathrm{H}$ NMR spectra of the derivatives $4 \mathbf{a}-1$ and 5 are observed in the region 2.85-2.89 ppm while the signal of $\mathrm{NCH}_{3}$ is shifted and, for compounds 4 , it is observed in the region 3.45-3.55 ppm. In the spectrum of the compound 5 , the signal of methylene protons is observed at $4.53 \mathrm{ppm}$, although for the compounds 4a-1 bearing acetamide substituents these signals are in the region 4.21-4.34 ppm. The acetamide NH signals are observed in the region 9.31-10.73 ppm; it should be mentioned that their position significantly depends on the type of amide substituent.

In the ${ }^{13} \mathrm{C}$ NMR spectra of the compounds $4 \mathrm{a}-1$ and 5 the signals of $\mathrm{CH}_{3}$ groups 14.7-15.4 (thiophene- $\left.\mathrm{CH}_{3}\right)$ Ta 30.4-30.6 ppm $\left(\mathrm{NCH}_{3}\right)$, which is almost similar to the positions of the signals for the same groups for the molecule of 314.4 Ta 33.0 ppm respectively. For most of the compounds 4, the signal of the $\mathrm{CH}_{2}$ Carbon atom is observed in the region 37.6-37.9 ppm; this signal slightly changes its position only for the derivatives 4c (2-ethtylphenyl amide) at 36.9 ppm, for 41 (2-aminothiazolyl amide) at 36.3 ppm and $4 \mathbf{i}$ (5-chloro-2-methylphenmyl amide) at $37.0 \mathrm{ppm}$ (probably due to either ortho-substitution or heterocycle), while compound 5 shows this signal at $36.3 \mathrm{ppm}$. It should be also mentioned that the spectra of compounds 4 and 5 are lacking the signal in the 174-175 ppm region, which is typical for $C=S$ Carbon and is present in the spectra of the compounds 2 and 3.

\subsection{Antimicrobial Activity}

According to the results of antimicrobial activity screening via agar diffusion test, all the studied compounds have shown antimicrobial and antifungal activity with the most pronounced influence on Gram-positive bacteria, i.e., S. aureus ATCC 25923 and B. subtilis ATCC 6633. It is worth mentioning that the studied tienopyrimidines have shown moderate activity in relation to the Gram-negative bacteria, but they were equal to the reference drug Streptomycine in relation to P. aeruginosa ATCC 27853 inhibition. The latter has been chosen as the reference drug for the experiment as a well-known and widely studied representative of aminoglycoside antibiotics with a broad spectrum of antibacterial activity, which is useful for experimental and clinical purposes [40-42]. Moreover, the aminoglycoside antibiotics were used as the reference drugs in the study of antibacterial activity spectrum for the selective inhibitors of TrmD [23], because among the TrmD inhibitors for now, there are not any approved and well-studied drugs with the confirmed mechanism of action on the market.

According to the results of antimicrobial activity screening (Table 2), the most active compound $\mathbf{4 d}$ with iso-propyl substituent at the aromatic amide ring was more active than the reference drug against Gram-positive bacteria and showed similar to its activity for Gram-negative strains; it also inhibited the growth of the C. albicans strain. 
Table 2. Screening data for antimicrobial activity of thieno[2,3-d]pyrimidine derivatives 3, 4a-1, 5 .

\begin{tabular}{|c|c|c|c|c|c|c|}
\hline \multirow{3}{*}{ Compounds * } & \multicolumn{6}{|c|}{ Average Diameter (mm) of Growth Inhibition Zone, Number of Test Repetitions $n=3$} \\
\hline & \multicolumn{2}{|c|}{ Gram-Positive Bacteria } & \multicolumn{3}{|c|}{ Gram-Negative Bacteria } & \multirow{2}{*}{$\begin{array}{c}\text { Fungus } \\
\text { C. albicans } \\
\text { ATCC } 653 / 885\end{array}$} \\
\hline & $\begin{array}{c}\text { S. aureus } \\
\text { ATCC } 25923\end{array}$ & $\begin{array}{c}\text { B. subtilis } \\
\text { ATCC } 6633\end{array}$ & $\begin{array}{c}E . \text { coli } \\
\text { ATCC } 25922\end{array}$ & $\begin{array}{l}\text { P. vulgaris } \\
\text { ATCC } 4636\end{array}$ & $\begin{array}{l}\text { P. aeruginosa } \\
\text { ATCC } 27853\end{array}$ & \\
\hline 3 & $25,25,25$ & $25,26,25$ & $22,21,21$ & $20,21,20$ & $22,21,21$ & $22,21,21$ \\
\hline $4 a$ & $23,23,23$ & $27,27,26$ & $21,21,21$ & $21,20,20$ & $21,21,21$ & $22,21,21$ \\
\hline $4 \mathrm{~b}$ & $20,21,20$ & $26,26,26$ & $20,21,21$ & $18,18,18$ & $19,20,20$ & $21,21,21$ \\
\hline $4 c$ & $22,21,21$ & $27,26,26$ & $20,20,20$ & $19,18,18$ & $21,21,21$ & $22,22,22$ \\
\hline $4 d$ & $25,26,25$ & $28,28,28$ & $23,24,24$ & $20,21,20$ & $22,21,22$ & $20,21,21$ \\
\hline $4 e$ & $25,25,25$ & $26,25,25$ & $23,21,22$ & $19,19,19$ & $22,21,21$ & $22,21,21$ \\
\hline $4 f$ & $24,24,24$ & $26,25,25$ & $22,22,22$ & $20,19,19$ & $22,21,21$ & $20,21,21$ \\
\hline $4 g$ & $22,23,23$ & $26,26,26$ & $23,23,23$ & $20,20,20$ & $20,21,21$ & $22,22,22$ \\
\hline $4 h$ & $25,26,25$ & $25,26,26$ & $23,24,24$ & $20,20,20$ & $21,21,21$ & $20,20,20$ \\
\hline $4 i$ & $23,23,23$ & $25,25,25$ & $22,20,21$ & $21,19,20$ & $21,21,21$ & $22,23,22$ \\
\hline $4 j$ & $23,23,23$ & $25,26,26$ & $22,21,21$ & $21,19,20$ & $20,20,20$ & $23,23,23$ \\
\hline $4 \mathrm{k}$ & $22,22,22$ & $27,27,27$ & $20,21,20$ & $20,19,20$ & $21,20,20$ & $21,21,21$ \\
\hline 41 & $20,21,20$ & $23,24 ., 23$ & $20,19,19$ & $18,18,19$ & $20,21,21$ & $21,21,21$ \\
\hline 5 & $22,23,23$ & $26,26,26$ & $22,22,22$ & $20,20,20$ & $20,21,20$ & $21,21,21$ \\
\hline Streptomycin & $26,25,25$ & $27,28,27$ & $25,25,25$ & $22,22,22$ & $22,21,21$ & $-* *$ \\
\hline
\end{tabular}

* The concentration of the compounds and the comparison drug streptomycin $100 \mu \mathrm{g} / \mathrm{mL} .{ }^{* *}$ Lack of antifungal activity.

In addition, the results of the antimicrobial activity study for the compound 3 (the activity of which was on a similar level with the other alkylated 4a-1 derivatives) assumes the pivotal role of 6-(benzimidazol-2-yl)-3,5-dimethyl-2-thioxo-thieno[2,3- $d$ ]pyrimidin-4-one structure in the realization of the antimicrobial activity.

As the resistance of microorganisms to many antibiotics (Streptomycin among them) develops $[43,44]$, the obtained results of antimicrobial activity screening and prediction of affinity to the binding site of selective inhibitors of TrmD for the novel benzimidazolethienopyrimidines shows that further studies of them as antibacterial agents can be considered promising.

\section{Conclusions}

The successful application of the hybrid pharmacophore approach allowed us to obtain the novel antimicrobial agents among the hybrids of thieno[2,3- $d$ ]pyrimidin-4ones with benzimidazole fragment at position 6. According to the docking studies results obtained for the benzimidazole-thienopyrimidines, they have a high affinity to the binding site of inhibitors of TrmD; this enzyme plays a crucial role in survival of most Gram-positive and Gram-negative bacteria, including P. aeruginosa (which is highly resistant to many known antibiotics). The optimal synthetic scheme for the preparation of 6-(1H-benzimidazol-2-yl)-3,5-dimethyl-2-thiothieno[2,3- $d$ ]pyrimidin-4-one with benzyl or the series of acetamide substituents attached to the Sulfur atom at position 2 of thienopyrimidine was developed. The antimicrobial activity screening results for the obtained compounds revealed their high level of growth inhibitory activity against Gram-positive bacteria (S. aureus and B. subtilis) and their moderate activity against Gram-negative bacterial strains with the activity to $P$. aeruginosa at the same level as that of the reference antibiotic. 2-\{[6-(1H-Benzimidazol-2-yl)-3,5-dimethyl-4-oxo-3,4-dihydrothieno[2,3- $d]$ pyrimidin-2yl]thio\}-N-(4-iso-propylphenyl)acetamide showed the highest in vitro antimicrobial activity among the tested compounds of the series; the same molecule has the highest affinity level to the active site of TrmD inhibitors isolated from $P$. Aeruginosa, according to the docking results. The obtained results can form the theoretical background for evaluation of the rationality of the further in vitro studies of the mechanism of antibacterial action of differently substituted derivatives of thieno[2,3- $d]$ pyrimidine. 
Author Contributions: Conceptualization, O.D.V., H.I.S., V.A.G.; Methodology and experimental work, O.D.V., K.Y.K., H.I.S., A.I.M.A.S., O.V.B.; Data Analysis, O.D.V., H.I.S., A.I.M.A.S., V.S.V.; writing-review and editing, O.D.V., H.I.S., O.V.B., V.A.G.; Project administration and Supervision, V.A.G., S.V.V., O.V.B. All authors have read and agreed to the published version of the manuscript.

Funding: Funding: The research was funded by the Ministry of Health Care of Ukraine at the expense of the State Budget in the framework \# 2301020 "Scientific and scientific-technical activity in the field of health protection" on the topic "Synthesis and study of new thienopyrimidines for the detection of antimicrobial and related types of pharmacological activity" (State registration number: 0121U109472. Order of the Ministry of Health of Ukraine of November 17, 2020 № 2651).

Institutional Review Board Statement: Not applicable.

Informed Consent Statement: Not applicable.

Data Availability Statement: The data presented in this study are available in this article.

Acknowledgments: The authors acknowledge Enamine Ltd. for the measurement of ${ }^{13} \mathrm{C}$ NMR and LC-MS spectra of the obtained substances. We are grateful to T.P. Osolodchenko (Mechnikov Institute of Microbiology and Immunology of the NAMS of Ukraine, Kharkiv, Ukraine) for her assistance in carrying out antimicrobial research of the compounds synthesized and her valuable help when discussing the results.

Conflicts of Interest: The authors declare no conflict of interest.

\section{References}

1. Kousovista, R.; Athanasiou, C.; Liaskonis, K.; Ivopoulou, O.; Karalis, V. Association of Antibiotic Use with the Resistance Epidemiology of Pseudomonas aeruginosa in a Hospital Setting: A Four-Year Retrospective Time Series Analysis. Sci. Pharm. 2021, 89, 13. [CrossRef]

2. Sirakanyan, S.N.; Geronikaki, A.; Spinelli, D.; Hakobyan, E.K.; Kartsev, V.G.; Petrou, A.; Hovakimyan, A.A. Synthesis and antimicrobial activity of new amino derivatives of pyrano $\left[4^{\prime \prime}, 3^{\prime \prime}: 4^{\prime}, 5^{\prime}\right]$ pyrido $\left[3^{\prime}, 2^{\prime}: 4,5\right]$ thieno[3,2-d]pyrimidine. An. Acad. Bras. Cienc. 2018, 90, 1043-1057. [CrossRef]

3. Sirakanyan, S.N.; Kartsev, V.G.; Geronikakim, A.; Spinellim, D.; Petrou, A.; Hakobyan, E.K.; Glamoclija, J.; Ivanov, M.; Sokovic, M.; Hovakimyan, A.A. Synthesis and Evaluation of Antimicrobial Activity and Molecular Docking of New N-1,3-thiazol2-ylacetamides of Condensed Pyrido[3',2':4,5] furo(thieno)[3,2-d]pyrimidines. Curr. Top. Med. Chem. 2020, 20, $2192-2209$. [CrossRef]

4. Kumari, M.A.; Triloknadh, S.; Harikrishna, N.; Vijjulatha, M.; Venkata Rao, C. Synthesis, antibacterial activity, and docking studies of 1,2,3-triazole-tagged thieno[2,3-d]pyrimidinone derivatives. J. Heterocycl. Chem. 2017, 54, 3672-3681. [CrossRef]

5. Khatri, T.T.; Shah, V.H. Effective microwave synthesis of bioactive thieno[2,3-d]pyrimidines. J. Chil. Chem. Soc. 2017, 62, 3354-3358. [CrossRef]

6. Vlasov, S.V.; Kovalenko, S.M.; Chernykh, V.P.; Krolenko, K.Y. Synthesis of 5-methyl-4-thio-6-(1,3,4-oxadiazol-2-yl)thieno[2,3d]pyrimidines and their antimicrobial activity study. J. Chem. Pharm. Res. 2014, 6, 22-27.

7. Vlasov, S.V.; Kovalenko, S.M.; Osolodchenko, T.P.; Vlasov, V.S. The study of the antimicrobial activity of the derivatives of 6-(1,2,4-oxadizol-3-yl)- and 6-(2-aminothiazol-4-yl)thieno[2,3-d]pyrimidin-4-ones by the double dilution method. J. Org. Pharm. Chem. 2019, 17, 26-30. [CrossRef]

8. Vlasov, S.V.; Kovalenko, S.N.; Osolodchenko, T.P.; Lenitskaya, E.B.; Chernykh, V.P. Synthesis and biological activity of 6-(1,3benzoxazol-2-yl)-5-methylthieno-[2,3-d]pyrimidines. Pharm. Chem. J. 2018, 52, 510-514. [CrossRef]

9. Settypalli, T.; Chunduri, V.R.; Kerru, N.; Nallapaneni, H.K.; Chintha, V.R.; Daggupati, T.; Yeguvapalli, S.; Wudayagiri, R. Design, synthesis, neuroprotective and antibacterial activities of 1,2,4-triazolo[3,4-b]1,3,4-thiadiazole linked thieno[2,3-d]pyrimidine derivatives and in silico docking studies. Chem. Select. 2019, 4, 1627-1634. [CrossRef]

10. Kerru, N.; Settypalli, T.; Nallapaneni, H.; Chunduri, V.R. Novel Thienopyrimidine derivatives containing 1,2,4-triazoles and 1,3,4-oxadiazoles as potent antimicrobial activity. Med. Chem. 2014, 4, 623-629. [CrossRef]

11. Triloknadh, S.; Rao, C.V.; Nagaraju, K.; Hari Krishna, N.; Ramaiah, C.V.; Rajendra, W.; Trinath, D.; Suneetha, Y. Design, synthesis, neuroprotective, antibacterial activities and docking studies of novel thieno[2,3-d]pyrimidine-alkyne Mannich base and oxadiazole hybrids. Bioorg. Med. Chem. Lett. 2018, 28, 1663-1669. [CrossRef]

12. Abbas, S.E.; Gawad, N.M.A.; George, R.F.; Akar, Y.A. Synthesis, antitumor and antibacterial activities of some novel tetrahydrobenzo [4, 5] thieno [2, 3-d] pyrimidine derivatives. Eur. J. Med. Chem. 2013, 65, 195-204. [CrossRef] [PubMed]

13. Mahmoud, M.R.; Abu El-Azm, F.S.M.; Ali, A.T.; Ali, Y.M. Design, synthesis, and antimicrobial evaluation of novel thienopyrimidines and triazolothienopyrimidines. Synth. Commun. 2015, 45, 982-992. [CrossRef]

14. Chambhare, R.V.; Khadse, B.G.; Bobde, A.S.; Bahekar, R.H. Synthesis and preliminary evaluation of some N-[5-(2-furanyl)-2methyl-4-oxo-4H-thieno[2,3-d]pyrimidin-3-yl]-carboxamide and 3-substituted-5-(2-furanyl)-2-methyl-3H-thieno[2,3-d]pyrimidin4-ones as antimicrobial agents. Eur. J. Med. Chem. 2003, 38, 89-100. [CrossRef] 
15. Prabhakar, V.; Kondra, S.B.; Maddula, S.R.; Parandhama, G.; Latha, J. Synthesis, structural elucidation of novel thieno[2,3-d]pyrimidine core unit containing 1,2,4-triazoles and thiophenes as potent antimicrobial activity. Org. Chem. Curr. Res. 2016, 5, 169. [CrossRef]

16. Saddik, A.A.; Kamal El-Dean, A.M.; El-Said, W.A.; Hassan, K.M.; Abbady, M.S. Synthesis, antimicrobial, and anticancer activities of a new series of thieno[2,3-d]pyrimidine derivatives. J. Heterocyclic. Chem. 2018, 55, 2111-2122. [CrossRef]

17. Vilchèze, C.; Baughn, A.D.; Tufariello, J.; Leung, L.W.; Kuo, M.; Basler, C.F.; Alland, D.; Sacchettini, J.C.; Freundlich, J.S.; Jacobs, W.R. Novel Inhibitors of InhA Efficiently Kill Mycobacterium tuberculosis under Aerobic and Anaerobic Conditions. Antimicrob. Agents Chemother. 2011, 55, 3889-3898. [CrossRef]

18. Li, S.-G.; Vilchèze, C.; Chakraborty, S.; Wang, X.; Kim, H.; Anisetti, M.; Ekins, S.; Rhee, K.Y.; Jacobs, W.R., Jr.; Freundlich, J.S. Evolution of a thienopyrimidine antitubercular relying on medicinal chemistry and metabolomics insights. Tetrahedron Lett. 2015, 56, 3246-3250. [CrossRef]

19. Harrison, G.A.; Mayer Bridwell, A.E.; Singh, M.; Jayaraman, K.; Weiss, L.A.; Kinsella, R.L.; Aneke, J.S.; Flentie, K.; Schene, M.E.; Gaggioli, M.; et al. Identification of 4-Amino-Thieno[2,3-d]Pyrimidines as QcrB Inhibitors in Mycobacterium tuberculosis. mSphere 2019, 4, e00606-19. [CrossRef]

20. Angula, K.T.; Legoabe, L.J.; Beteck, R.M. Chemical Classes Presenting Novel Antituberculosis Agents Currently in Different Phases of Drug Development: A 2010-2020 Review. Pharmaceuticals 2021, 14, 461. [CrossRef]

21. Zhong, W.; Koayc, A.; Ngoc, A.; Lic, Y.; Naha, Q.; Wong, Y.H.; Chionh, Y.H.; Ngc, H.Q.; KohStentac, X.; Poulsenc, A.; et al. Targeting the bacterial epitranscriptome: Discovery of novel tRNA-(N1G37) methyltransferase (TrmD) inhibitors with antibiotic activity. ACS Infect. Dis. 2019, 5, 326-335. [CrossRef]

22. Hill, P.J.; Abibi, A.; Albert, R.; Andrews, B.; Gagnon, M.M.; Gao, N.; Grebe, T.; Hajec, L.I.; Huang, J.; Livchak, S.; et al. Selective inhibitors of bacterial t-RNA-(N(1)G37) methyltransferase (TrmD) that demonstrate novel ordering of the lid domain. J. Med. Chem. 2013, 56, 7278-7288. [CrossRef] [PubMed]

23. Zhong, W.; Pasunooti, K.K.; Balamkundu, S.; Wong, Y.H.; Nah, Q.; Gadi, V.; Gnanakalai, S.; Chionh, Y.H.; McBee, M.E.; Gopal, P.; et al. Thienopyrimidinone derivatives that inhibit bacterial tRNA (guanine37-N1)-methyltransferase (TrmD) by restructuring the active site with a tyrosine-flipping mechanism. J. Med. Chem. 2019, 62, 7788-7805. [CrossRef]

24. Alasmary, F.A.S.; Snelling, A.M.; Zain, M.E.; Alafeefy, A.M.; Awaad, A.S.; Karodia, N. Synthesis and evaluation of selected benzimidazole derivatives as potential antimicrobial agents. Molecules 2015, 20, 15206-15223. [CrossRef]

25. Picconi, P.; Hind, C.; Jamshidi, S.; Nahar, K.; Clifford, M.; Wand, M.E.; Sutton, J.M.; Rahman, K.M. Triaryl benzimidazoles as a new class of antibacterial agents against resistant pathogenic microorganisms. J. Med. Chem. 2017, 60, 6045-6059. [CrossRef]

26. Antoci, V.; Cucu, D.; Zbancioc, G.; Moldoveanu, C.; Mangalagiu, V.; Amariucai-Mantu, D.; Aricu, A.; Mangalagiu, I. Bis(imidazole/benzimidazole)-pyridine derivatives: Synthesis, structure and antimycobacterial activity. Future Med. Chem. 2020, 12, 207-222. [CrossRef]

27. Al-Harthy, T.; Al-Sadi, A.M.; Zoghaib, W.; Moghadam, E.S.; Stoll, R.; Abdel-Jalil, R. Design, Synthesis and Bioactivity of Benzimidazole-2-Carbamates as Soil-Borne Anti-Fungal Agents. Chem. Proc. 2021, 3, 64. [CrossRef]

28. Pereira, G.A.; Santos, L.H.; Wang, S.C.; Martins, L.C.; Villela, F.S.; Liao, W.; Dessoy, M.A.; Dias, L.C.; Andricopulo, A.D.; Costa, M.A.; et al. Benzimidazole inhibitors of the major cysteine protease of Trypanosoma brucei. Future Med. Chem. 2019, 11, 1537-1551. [CrossRef]

29. Yoon, Y.K.; Ali, M.A.; Wei, A.C.; Choon, T.S.; Ismail, R. Synthesis and evaluation of antimycobacterial activity of new benzimidazole aminoesters. Eur. J. Med. Chem. 2015, 93, 614-624. [CrossRef] [PubMed]

30. Miller, S.I. Antibiotic resistance and regulation of the gram-negative bacterial outer membrane barrier by host innate immune molecules. mBio 2016, 7, me01541-16. [CrossRef]

31. Mookherjee, P.; Green, P.S.; Watson, G. AutoDock4 and AutoDockTools4: Automated docking with selective receptor flexibility. J. Comput. Chem. 2009, 30, 2785-2791. [CrossRef]

32. Protein Data Bank. Available online: http://www.rcsb.org/pdb/home/home.do (accessed on 4 April 2020).

33. Trott, O.; Olson, A.J. AutoDock Vina: Improving the speed and accuracy of docking with a new scoring function, efficient optimization, and multithreading. J. Comput. Chem. 2010, 31, 455-461. [CrossRef]

34. Vlasov, S.V.; Chernykh, V.P.; Osolodchenko, T.P. Synthesis and the antimicrobial activity of ethyl 3-alkyl-2-(alkylthio)-5-methyl-4oxo-3,4-dihydrothieno[2,3-d]pyrimidine-6-carboxylate derivatives. News Pharm. 2015, 3, 3-8. [CrossRef]

35. Clinical and Laboratory Standards Institute (CLSI). Performance Standards for Antimicrobial Susceptibility Testing, 27th ed.; CLSI supplement M100-S26; CLSI: Wayne, PA, USA, 2017; p. 280.

36. Ministry of Public Health of Ukraine. Bacteriological Control of Culture Media; Newsletter № 05.4.1/1670; Ministry of Public Health of Ukraine: Kiev, Ukraine, 2001; p. 45.

37. Nekrasova, L.S.; Svita, V.M.; Glushkevich, T.G.; Tomchuk, V.V.; Zherebko, N.M.; Yanovs'ka, V.V. Methodological Guidelines "Determination of the Sensitivity of Microorganisms to Antibiotics"; № MB 9.9.5-143-2007; Ministry of Public Health of Ukraine: Kiev, Ukraine, 2007; p. 24.

38. Baber, J.C.; Thompson, D.C.; Cross, J.B.; Humblet, C. GARD: A generally applicable replacement for RMSD. J. Chem. Inf. Model. 2009, 49, 1889-1900. [CrossRef] [PubMed]

39. Vlasov, S.V.; Chernykh, V.P. Synthesis, antiinflammatory and antimicrobial activity of 6-(1H-benzimidazol-2-yl)-5-methyl-4(alkylthio)thieno[2,3-d]pyrimidines. News Pharm. 2016, 3, 9-16. [CrossRef] 
40. Vianna, J.F.; Bezerra, K.S.; Oliveira, J.I.N.; Albuquerque, E.L.; Fulco, U.L. Binding energies of the drugs capreomycin and streptomycin in complex with tuberculosis bacterial ribosome subunits. Phys. Chem. Chem. Phys. 2019, 21, 19192-19200. [CrossRef] [PubMed]

41. Germovsek, E.; Barker, C.I.; Sharland, M. What do I need to know about aminoglycoside antibiotics? Arch. Dis. Child. Educ. Pract. Ed. 2017, 102, 89-93. [CrossRef]

42. Li, R.; Yuan, X.; Wei, J.; Zhang, X.; Cheng, G.; Wang, Z.A.; Du, Y. Synthesis and Evaluation of a Chitosan OligosaccharideStreptomycin Conjugate against Pseudomonas aeruginosa Biofilms. Mar. Drugs 2019, 17, 43. [CrossRef] [PubMed]

43. Peterson, E.; Kaur, P. Antibiotic resistance mechanisms in bacteria: Relationships between resistance determinants of antibiotic producers, environmental bacteria, and clinical pathogens. Fron. Microbiol. 2018, 9, 2928. [CrossRef]

44. Rodríguez-García, A.; Mares-Alejandre, R.E.; Muñoz-Muñoz, P.L.A.; Ruvalcaba-Ruiz, S.; González-Sánchez, R.A.; Bernáldez-Sarabia, J.; Meléndez-López, S.G.; Licea-Navarro, A.F.; Ramos-Ibarra, M.A. Molecular analysis of Streptomycin resistance genes in clinical strains of Mycobacterium tuberculosis and biocomputational analysis of the MtGidB L101F variant. Antibiotic 2021, 10, 807. [CrossRef] 